Volume 3, Issue 9, September-2016, pp. 482-486

\title{
Mitigation of ICl Through Optimized SSR ICI Self Cancellation Scheme in OFDM
}

\author{
${ }^{1}$ Shyam Sunder Kongonda , ${ }^{2}$ Dr.Srinivasulu Tadishetty \\ ${ }^{1}$ M.Tech Digital Communications and ${ }^{2}$ Professor, E.C.E. Department \\ ${ }^{1,2}$ Kakatiya University College of Engineering and Technology, Warangal, India \\ ${ }^{1}$ shyamsunder429@gmail.com ,2drstadishetty@gmail.com
}

\begin{abstract}
Orthogonal frequency division multiplexing (OFDM) is extensively used in wireless communication systems because it offers high data rates and provides a better solution to inter symbol interference (ISI) caused by a multipath channel. A well known problem of OFDM system is sensitive to carrier frequency offset $(\delta)$ between the transmitted and received signals. The Frequency offset in wireless communication systems ruins the Orthogonality between subcarriers resulting in Inter Carrier Interference (ICI).Which is a severe problem in OFDM system and eventually decrease the performance of the system. There are numerous techniques are in existence to mitigate the effect of $\mathrm{ICl}$ caused by CFO (carrier frequency offset). This paper investigates an innovative ICI cancellation method termed as optimized SSR (symmetric symbol repeat) ICI self cancellation scheme. The proposed method utilizes an optimum data allocation $(1,-\lambda)$ and combining $(1,-\mu)$ scheme to improve CIR performance for an estimated normalized frequency offset $\delta$. However, this requires continuous CFO estimation and feedback circuitry. To avoid the requirement of CFO, sub optimal values ( $\lambda$ so, $\mu$ so) are considered for combating the effect of $\mathrm{ICl}$. This paper mainly focus on comparison of proposed method with the previous reduction methods and standard OFDM system in terms of CIR (carrier to interference) \& BER (bit error rate) performance.
\end{abstract}

Keywords: OFDM, ICI, ISI, CFO, CIR, BER, QAM

\section{I.INTRODUCTION}

In Today's world OFDM plays vital role in wireless communication systems, which is a promising modulating technique for high data rate transmission in wireless communication [1] systems. OFDM is a form of multi-carrier modulation technique [2], which effectively utilizes the spectrum by spacing the channels much closer together. This spectral efficiency can be achieved by making orthogonality between the sub carriers and preventing interference between the subcarriers. In OFDM, a high-rate data stream is divided into many lowerrate streams which are mapped (by using QAM or QPSK) onto mutually overlapping subcarriers and transmitted simultaneously i.e., high rate bit stream is split into $\mathrm{N}$ parallel lower rate bit streams and each of these streams are modulated using one of $\mathrm{N}$ orthogonal sub-carriers. However, OFDM is being widely used in many applications such as IEEE 802.11a, the multimedia mobile access communication (MMAC), HIPERLAN/2, Digital Video Broadcasting (DVB) and so on.. In a basic communication system, the input data is modulated using only one carrier frequency. Therefore the available channel bandwidth is totally occupied by each symbol. This type of mechanism can lead to inter symbol-interference (ISI) in frequency selective channels.

By inserting Guard interval with the cyclic extension of the OFDM symbol, the inter symbol interference (ISI) can be easi- ly avoided. A well known problem with OFDM is its vulnerability to slight differences in frequency at the transmitter and receiver, normally referred as frequency offset, caused by Doppler shift due to relative motion between the transmitter and receiver [3]. This carrier frequency offset (CFO) causes distorts orthogonality between sub-carriers, which implies inter-carrier interference (ICI). There is one more deleterious effect caused by frequency offset is the reduction of signal amplitude or peak in the output of the filters. The unwanted ICI causes leakage of power among subcarriers and it eventually degrades the Bit error Rate (BER) performance of the OFDM receiver. In the presence of ICI, it is very difficult to obtain the theoretical BER and CIR. The BER of the received signals are very sensitive to Doppler effects and frequency offsets.

\section{II.LITERATURE SURVEY}

\section{OFDM}

In an OFDM system, the input bit data stream is multiplexed into $\mathrm{N}$ symbol streams, each with $\mathrm{T}$ symbol period and each stream is modulated parallelly on synchronous sub-carriers. The discrete time OFDM symbol after the IFFT block at the transmitter can be written as 
Shyam Sunder Kongonda and Dr.Srinivasulu Tadishetty," Mitigation of ICI Through Optimized SSR ICI Self Cancellation Scheme in OFDM", International Journal of Computer Engineering In Research Trends, Volume 3, Issue 9, September-2016, pp. 482-486

$x[n]=\frac{1}{\sqrt{N}} \sum_{k=0}^{N-1} X(K) e^{\frac{j 2 \pi n k}{N}}, n=0,1,2 \ldots N-1$

Where $\mathrm{N}$ is no. of subcarriers and $\mathrm{X}(\mathrm{k})$ denotes the modulated data symbol transmitted (M-ary phase-shift keying :BPSK,QPSK,QAM) on $\mathrm{k}^{\text {th }}$ subcarrier. Due to frequency offset and AWGN channel, the received OFDM signal can be expressed as

$$
y[n]=x[n] e^{\frac{j 2 \pi \delta n}{N}}+z[n], n=0,1,2 \ldots N-1
$$

Where $\delta$ represents the frequency offset obtained by calculating the frequency spacing between two subcarriers and $\mathrm{z}[\mathrm{n}]$ is a zero-mean AWGN. The frequency domain signal at the $\mathrm{k}^{\text {th }}$ subcarrier with frequency offset after the Fast Fourier transform (FFT) block is given as

$$
Y[k]=X[k] S(0)+\sum_{m=0, m \neq k}^{N-1} X(m) S(m-k)+Z(k)
$$

Where $Z(k)$ is $k^{\text {th }}$ sample DFT of $z(n)$ and the term $S(m-k)$ is the ICI weighting coefficient between $\mathrm{k}^{\text {th }}$ and $\mathrm{m}^{\text {th }}$ subcarriers, which is given as

$S(m-k)=e^{\left(j \pi(m+\delta-k)\left(1-\frac{1}{N}\right)\right.} \frac{\sin (\pi(m+\delta-k))}{N \sin \left(\frac{\pi}{N}(m+\delta-k)\right)}$

Carrier-to-Interface Ratio (CIR) is defined as the ratio of the desired signal power to the power in the interference component. It serves as a good indication of signal quality. A channel with constant CFO, CIR is improved more than $15 \mathrm{~dB}$ for group size is two. The desired signal is transmitted on at $\mathrm{k}^{\text {th }}$ sub carrier, then the CIR of Normal OFDM systems is simplified as:

$$
C I R=\frac{E\left[|C(k)|^{2}\right]}{E\left[|I C I(k)|^{2}\right]}=\frac{|S(0)|^{2}}{\sum_{m=1}^{N-1}|S(m)|^{2}}
$$

CIR is a function of $\mathrm{N}$ and $\delta$.

\section{PHASE SHIFT KEYING:}

The M-PSK modulation technique includes BPSK, QPSK, 16PSK. At the transmitter section, the input bit data is first encoded by the channel to decrease the probability of error at the receiver.

In BPSK constellation $\mathrm{dmin}=2 \mathrm{~A}$ and $\mathrm{rb}$ is defined as $\mathrm{Eb}=\mathrm{N} 0$ and sometimes it is called SNR per bit. With this definition we have:

$$
\mathrm{rb}=\frac{\mathrm{Eb}}{\mathrm{N} 0}=\frac{\mathrm{dmin}^{2}}{4 \mathrm{~N} 0}
$$

For BPSK modulation technique, the theoretical equation for bit error rate (BER) in Additive White Gaussian Noise (AWGN) channel is

$$
\mathrm{Pb}=\mathrm{Q}(\sqrt{2 \mathrm{rb}})
$$

Where $\mathrm{Q}$ is a Gaussian function.

For MPSK signaling, we can easily calculate an approximation of BER using nearest neighbor approximation. The bit error probability can be approximated by:

$$
\mathrm{Pb}=\frac{2}{\log _{2} \mathrm{M}} \mathrm{Q}\left(\sqrt{2 \gamma \mathrm{b} \log _{2} \mathrm{M}} \sin \left(\frac{\pi}{M}\right)\right.
$$

This approximation is only good for high SNR.

$\boldsymbol{Q A M}$ is a combination of amplitude and phase shift keying, which is extensively used as a modulation technique in digital telecommunication system. In QAM, high spectral efficiencies can be achieved by considering a suitable constellation size.

Bit error probability for MQAM can be approximated as

$\mathrm{Pb}=\frac{4}{\log _{2} \mathrm{M}} \mathrm{Q}\left(\sqrt{\frac{3 r \mathrm{~b} \log _{2} \mathrm{M}}{\mathrm{M}-1}}\right)$

\section{Advantages of MQAM over MPSK}

i. FOR MPSK, the signal canstellation is circular, where as for M.QAM it is rectangular.

ii. The bandwidth requirement of QAM is same as MPSK, but dMQAM>dMPSK for same transmitted power. Hence, QAM gives superior performance than that of MPSK.

iii. superior performance of MQAM can be realized only if the channel is free from nonlineari$\operatorname{ty}(\therefore$ amplitude variation is required for M-QAM)

\section{ICI REDUCTION METHODS}

Several ICI self cancellation methods were proposed in the literature during last few decades.

\section{A. ICI Self Cancellation Method}

ICI self cancellation scheme proposed by Zhao utilizes data allocation and combining of $(1,-1)$ on two adjacent subcarriers [4] i.e., same data is modulated at $k^{\text {th }}$ and $\mathrm{k}+1^{\text {th }}$ subcarriers using $(1,-1)$ as data allocation and are grouped at the receiver with coefficient weights 1 and -1 . However, its performance degrades at higher values of $\delta$.

\section{B. Conjugate Cancellation}

Conjugate Cancellation had been proposed by Yeh, Chang and Hassibi. In this scheme, OFDM signal \& its conjugate are multiplexed, transmitted and grouped at the receiver to reduce the effect of ICI [5]. However, its performance decreases as $\delta$ increases. At higher $\delta>0.25$, its CIR performance is aggravate than standard OFDM system.

\section{Phase Rotated Conjugate Cancellation (PRCC)}

Phase Rotated Conjugate Cancellation (PRCC) is an extension to conjugate cancellation, in which the phase is multiplied with the generated symbol and its conjugate signal to be transmitted over multiple paths [6]. Here the phase depends on $\delta$ and hence it needs continuous (CFO) estimation and feedback circuitry, which ultimately increases the complexity of the hardware.

\section{Symmetric Symbol Repeat ICI Self Cancellation} This method proposed by Sathanantham, Rajatheva and Slimane, which utilizes data allocation and combining of (1,- 
1) at $k^{\text {th }}$ and $(\mathrm{N}-1-\mathrm{k})^{\text {th }}$ subcarrier [7]. This scheme shows better CIR performance than ICI self cancellation scheme.

For the majority of subcarriers the difference between symmetrically placed ICI components is small (almost negligible). Therefore if a complex data pair ex: $(a,-a)$ is modulated on a pair of two symmetrically placed subcarriers, then the ICI generated by these orthogonal subcarriers will be cancelled out. In this method the data symbol to be transmitted at the $\mathrm{k}^{\text {th }}$ subcarrier is repeated at $(\mathrm{N}-1-\mathrm{k})^{\text {th }}$ the subcarrier with opposite polarity [8] i.e.,

$X(N-1)=-X(0), \ldots . X(N-1-k)=-X(k)$

The received data signal at the $\mathrm{k}^{\text {th }}$ subcarrier and $(\mathrm{N}-1-\mathrm{k})^{\text {th }}$ subcarrier is given by

$Y^{\prime}(k)=\sum_{m=0}^{\frac{N}{2}-1} X(m)(S(m-k)-S(m+1-k))+Z(k)$

$\left.Y^{\prime}(N-1-k)\right)=\sum_{m=0}^{\frac{N}{2}-1} X(m)(S(m+k+1-N)-$

$S(k-m))+Z(N-1-k)$

Where the ICI coefficient is given by

$S^{\prime}(m-k)=S(m-k)-S(m+1-k)$

At the receiver section, each signal at the $\mathrm{N}-1-\mathrm{k}^{\text {th }}$ subcarrier is multiplied by " -1 " and then added with the one at the $\mathrm{k}^{\text {th }}$ subcarrier

$\left.Y^{\prime \prime}(k)=Y^{\prime}(k)-Y^{\prime}(N-1-k)\right)$

$Y^{\prime \prime}(k)=\sum_{m=0}^{\frac{N}{2}-1} X(m)[(S(m-k)-S(N-1-m-k)-$

$S(m+1+k-N)+S(k-m))+Z(k)-Z(N-1-$

k) $; k=0,1, \ldots . \frac{N}{2}-1$

Thus CIR of conventional SSR ICI self cancellation scheme can be written as

$\mathrm{CIRc}=\frac{|2 S(0)-S(-N-1-2 k)-S(1-N+2 k)|^{2}}{\sum_{m=0, m \neq k}^{\frac{N}{2}-1}|S(m-k)-S(N-1-m-k)-S(m+k+1-N)+S(k-m)|^{2}}$

\section{PROPOSED METHOD}

\section{System model}

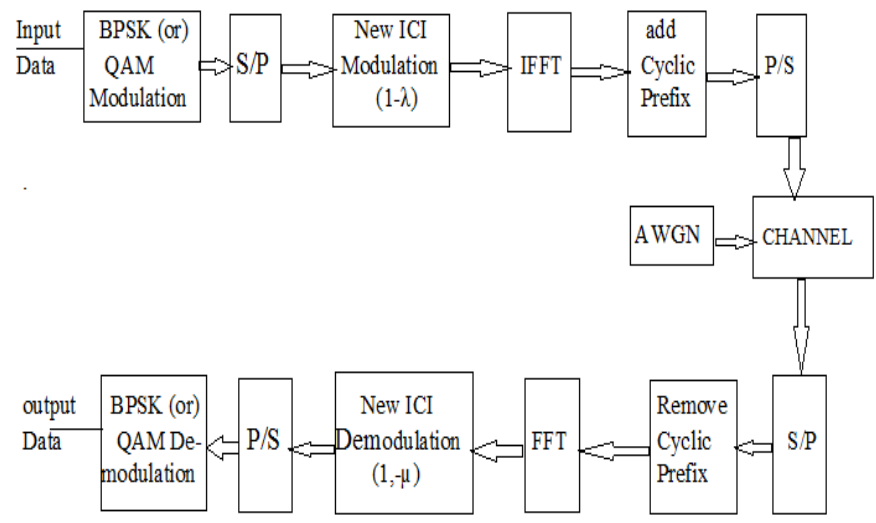

Fig 1: Block diagram of proposed scheme This paper deals with the improvement of previous methods and this method is an extension of SSR ICI Self-Cancellation technique.

Aim of the system model is to reduce the effect of ICI on OFDM by ICI modulation and ICI demodulation processes, without increasing the hardware complexity while maintaining bandwidth efficiency. The system model comprises of an OFDM transreceiver that works in following steps: BPSK (or) QAM, Serial to Parallel Conversion, ICI SC Modulation, IFFT, Add CP, parallel to serial converter followed by channel, Remove CP, FFT, ICI SC demodulation and BPSK (or) QAM demodulation.

In general at the transmitter a data allocation $(1,-\lambda)$ is used at symmetrical placed subcarriers i.e.,

$X(N-1)=-\lambda X(0) \ldots, X(N-1-k)=-\lambda X(k)$

Now the received data at the $\mathrm{k}^{\text {th }}$ subcarrier and $(\mathrm{N}-1-\mathrm{k})^{\text {th }}$ subcarrier is

$$
\begin{aligned}
& Y^{\prime}(k)=\sum_{m=0}^{\frac{N}{2}-1} X(m)(S(m-k)-\lambda S(m+1-k))+Z(k) \\
& \left.Y^{\prime}(N-1-k)\right)=\sum_{m=0}^{\frac{N}{2}-1} X(m)(S(m+k+1-N)- \\
& \lambda S(k-m))+Z(N-1-k))
\end{aligned}
$$

So the received data with combination of $\mathrm{k}^{\text {th }}$ and $(\mathrm{N}-1-\mathrm{k})^{\text {th }}$ subcarriers with $(1,-\mu)$

$$
Y^{\prime \prime}(k)=Y^{\prime}(k)-\mu Y^{\prime}(N-1-k)
$$

$Y^{\prime \prime}(k)=\sum_{m=0}^{\frac{N}{2}-1} X(m)[(S(m-k)-\lambda S(N-1-m-k)-$ $\mu S(m+1+k-N)+\lambda \mu S(k-m))+Z(k)-\mu Z(N-1-$ k) $\quad ; \quad k=0,1, \ldots \cdot \frac{N}{2}-1$

Thus the CIR of the proposed scheme is

$$
C I R p=\frac{|-\mu S(-N-1-2 k)+(1+\lambda \mu) 2 S(0)-\lambda S(1-N+2 k)|^{2}}{\sum_{m=0, m \neq k}^{\frac{N}{2}-1}|-\mu \lambda S(m-k)-\lambda S(N-1-m-k)-\mu S(m+k+1-N)+S(k-m)|^{2}}
$$

The optimal values $\lambda$ and $\mu$ have been found by using a technique known as Nelder Mead Simplex Algorithm (NMSA) [9]. These optimal values are calculated for at a very small interval of which results in maximize the CIR for given $\delta$. But the drawback is for every $\delta$, there are unique optimal values and always this needs a continuous CFO estimation, which increases the system complexity. For each pair of optimal values the CIR has been calculated which forms a matrix [8] i.e., the elements in the matrix as the form like CIRp $(\delta v, \lambda o v, \mu o v)$

$$
\text { Where } \mathrm{v}=\frac{\delta H-\delta L}{\nabla \delta}+1
$$

$\delta_{H}$ and $\delta_{L}$ are highest and the lowest possible values respectively and $\nabla \delta$ is normalized frequency offset. Here $\delta H$ $=0.25$ and $\delta L=0.03$ and $\nabla \delta=0.02$ so that $\mathrm{v}=12$ to avoid the above drawbacks sub-optimal pair $(\lambda s o, \mu s o)$ is considered by using the following criterion 
Shyam Sunder Kongonda and Dr.Srinivasulu Tadishetty," Mitigation of ICI Through Optimized SSR ICI Self Cancellation Scheme in OFDM", International Journal of Computer Engineering In Research Trends, Volume 3, Issue 9, September-2016, pp. 482-486

$$
\lambda s o, \mu s o=\max _{\lambda o, \mu o}\left[p-\frac{\sum_{j=1}^{p}(p-C I R(\delta \mathrm{j}, \lambda \mathrm{o}, \mu \mathrm{o}))}{v}\right]
$$

Where $\mathrm{p}$ is the maximum CIR of a particular row of the matrix and second term is mean deviation of the CIR of that row from the peak (p) of that row, here these sub-optimal values $(\lambda$ so, $\mu s o)$ are independent of $\delta$ in the proposed method. These values are. $\lambda s o=0.6164$ and $\mu s o=1.0351$

\section{IV.SIMULATION RESULTS}

Here we have considered an OFDM system with $\mathrm{N}=64$ subcarriers and BPSK or QAM technique is used to modulate each of the subcarrier. The simulation using MATLAB software are performed to evaluate CIR and BER performance The proposed scheme cancels the ICI coefficient effectively and further improves the system throughput performance as shown through extensive simulations.

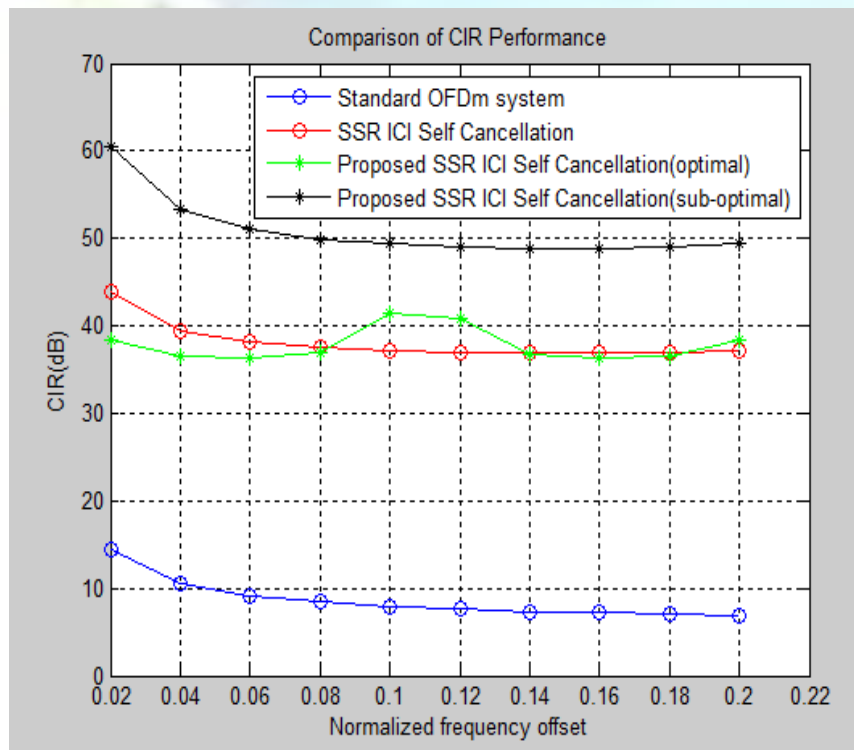

Fig 2: comparison of CIR performance

Analysis: The performance comparison results shows that proposed technique has better BER and improved CIR than standard OFDM and existing SC scheme. In this method the CIR performance is more than $25 \mathrm{~dB}$ is improved compared to standard OFDM for Small values of CFO ( $\delta)$.

For the proposed scheme CIR performance is slightly aggravate than conventional scheme $\delta \in(0.003,0.008)$

Analysis of BER: The BER performance of the proposed method is very much improved in comparison to standard OFDM system and very close to conventional SSR ICI self cancellation scheme.

However, the proposed scheme is little less efficient than conventional SSR ICI self cancellation in terms of BER

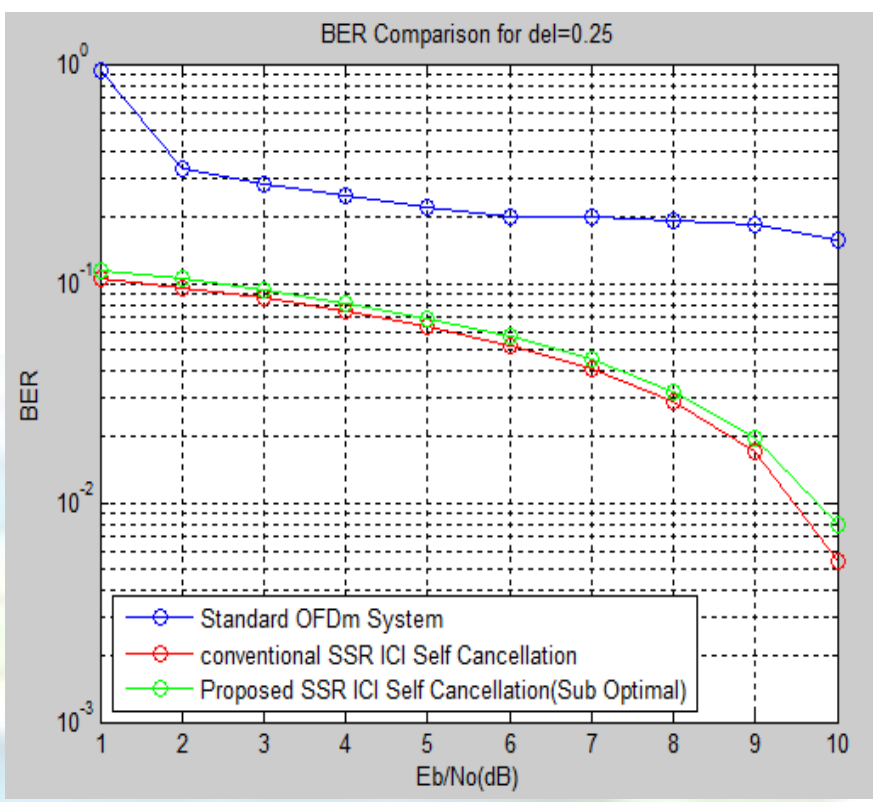

Fig 3: comparison of BER performance

\section{CONCLUSION}

In this paper several ICI minimization methods are studied and reviewed. It will be shown that our approach gives better results than previous reduction methods. Moreover, we propose to perform the signal processing only on the transmitter side and allowing a reduction of the receiver complexity without any performance degradation. The proposed technique effectively alleviates the effects of ISI, ICI and improves CIR performance.

\section{REFERENCES:}

[1] Y.Wu and W. Y. Zou, "Orthogonal frequency division multiplexing: A multicarrier modulation scheme", IEEE Transaction on Consumer Electronics, vol. 41, no. 3,pp 392-399, Aug. 1995

[2] R. van Nee and R. Prasad, OFDM for Wireless Multimedia Communications. Norwood, MA: Artech House, 2000

[3] P.H.Moose, "A Technique for orthogonal frequency division multiplexing frequency offset correction,", IEEE Trans. Commun., vol.42, pp2908-2914, Oct. 1994.

[4] Zhao Y, Haggman SG. "Inter carrier Interference SelfCancellation Scheme for OFDM Mobile Communication Systems", IEEE Trans.Commun 2001; 49(7): 1185-1191

[5] Yeh HG, Chang YK, Hassibi B. "A scheme for cancelling inter carrier interference using conjugate transmission in multicarrier communication systems", IEEE Trans. Wireless 
Shyam Sunder Kongonda and Dr.Srinivasulu Tadishetty," Mitigation of ICI Through Optimized SSR ICI Self Cancellation Scheme in OFDM", International Journal of Computer Engineering In Research Trends, Volume 3, Issue 9, September-2016, pp. 482-486

Communication 2007; 6(1): 3-7.

[6] Wang C-L, Huang Y-C. "Inter carrier Interference Cancellation Using General Phase Rotated Conjugate Transmission for OFDM Systems", IEEE Transactions on Communications 2010; 58(3): 812-819.

[7] K.Sathanantham, R.M.A.P.Rajatheva and Slimane Ben Slimane "Analysis of OFDM in the Presence of Frequency Offset and a Method to Reduce Performance Degradation" Global Tele communications Conference, 2000. GLOBECOM '00. IEEE,vol. 1,pp. 72-76, 2000

[8] Ashish goel, ankit nagpal and jasmeet kaur, "Optimized data allocation and combining scheme for ICI self cancellation in OFDM system" IEEE 978-1-4673-1989-8/12, Aug 2012.

[9] J. H. Mathews, K. K. Fink, "Numerical Methods using Mat lab', 4thedition, Prentice-Hall Inc. , 2004. 